\title{
Body surface area estimation: Cross validation of several prediction equations
}

\author{
V P Wickramasinghe ${ }^{1}$, G Sathyadas ${ }^{2}$, S P Lamabadusuriya ${ }^{1}$
}

Sri Lanka Journal of Child Health, 2009; 38: 54-59

(Key words: Body surface area, Sri Lankan children, Mosteller equation, Boyd-West normogram)

\begin{abstract}
Background Body surface area (BSA) is used in clinical practice to prescribe medication, standardize measured parameters etc. Normogram has been the gold standard. However, there are several prediction equations in the published literature. A simple method giving an accurate estimation would help to assess BSA in day to day clinical practice efficiently.
\end{abstract}

Objective To assess the accuracy of different equations for the estimation of BSA in a group of Sri Lankan children.

Method Data collected in 3 different school surveys carried out between 2002 and 2005 were used. BSA estimated by 9 prediction equations described in the literature were compared with the BSA assessed by normogram.

Results Two thousand three hundred and thirty eight girls and 639 boys, between the ages of 5-16 years, were assessed. The mean ages of the boys and girls were $10.3 \pm 1.47$ years and $11.5 \pm 2.47$ years respectively. The highest mean BSA estimation was given by Gehan \& George equation (boys $1.14 \pm 0.20 \mathrm{~m}^{2}$ and girls $1.2 \pm 0.25 \mathrm{~m}^{2}$ ) and lowest by Yu et al (boys $1.08 \pm 0.20 \mathrm{~m}^{2}$ and girls $1.13 \pm 0.25 \mathrm{~m}^{2}$ ). Estimates correlated highly with BSA assessed by normogram. When the estimates were compared with normogram, equation of Mattar gave the highest bias for both groups (females $0.0152 \mathrm{~m}^{2}$ and males $\left.0.0177 \mathrm{~m}^{2}\right)$. Mosteller equation gave relatively low bias (females $0.0077 \mathrm{~m}^{2}$, and males $0.0072 \mathrm{~m}^{2}$ ).

Conclusions All equations gave very good agreement between each other. Out of the equations, easiest to use in day to day clinical practice is the equation

${ }^{1}$ Department of Paediatrics, Faculty of Medicine, University of Colombo, ${ }^{2}$ Senior Registrar, Lady Ridgeway Hospital for Children, Colombo.

(Received on 7 November, 2008. Accepted 20 January 2009) described by Mosteller. The maximum bias in estimation would lead to less than $5 \%$ error in determining drug doses which is negligible in day to day clinical practice. Mosteller equation is suitable for routine clinical use.

\section{Introduction}

Body surface area (BSA) is extensively used in clinical practice either for normalizing physiological parameters such as cardiac output or renal clearance or in determining appropriate drug dose. BSA is estimated using height and weight. The established method of doing this is by using the normogram. However, there have been a few drawbacks in this method viz. the normogram chart is needed and it has been shown to have a higher degree of error in estimation of $\mathrm{BSA}^{1}$. There are numerous mathematical equations in the published literature, the main drawback being that they have complex calculations not suitable for day to day clinical practice even with the use of a simple calculator.

There is no gold standard for estimation of BSA. However the widespread use of Boyd-West normogram in clinical practice can be taken as a clinical standard ${ }^{1}$. The equation derived by $\mathrm{Yu}$ et al is considered as one of latest and has used 3dimensional one-pass whole body scanning ${ }^{2}$. A previous validation study in adults showed that $\mathrm{Yu}$ et al equation gave the lowest estimation ${ }^{2}$. For many years DuBios-DuBios equation was considered as one of the standards ${ }^{3}$. However, it was derived from a data set of nine subjects only ${ }^{4}$. Only Mosteller and Mattar equations had simplified modes of calculation. However, the accuracy of its use on children needs to be validated against a standard method.

This study was designed to compare the BSA assessed using different equations and BSA assessed by Boyd-West normogram. 


\section{Method}

Data collected in 3 different school surveys carried out between 2002 and 2005 among 5-16 year old healthy children was used. Height was measured to the closest $0.1 \mathrm{~cm}$ using a stadiometer (Surgical and Medical products, Australia) and weight was measured with minimum lightweight clothes to the closest 100g (Soehnle ${ }^{\circledR}$, Soehnle-Waagen $\mathrm{GmbH} \&$ Co, Germay). BSA was estimated by nine prediction equations (annexure) described in the literature. Boyd-West normogram was used as the standard and each individual's BSA was assessed using the normogram. Two authors (VPW and GS) made the calculations independently and compared. Any discrepancies were reassessed and mutually agreed on the final value.

Bias in the assessment of BSA was calculated by subtracting the BSA assessed by the prediction equation from the BSA assessed by the Boyd-West normogram. Bland Altman plot ${ }^{5}$ was used to compare the bias (predicted - actual) with the mean of the BSA calculated using each equation and the normogram. Correlation between bias and the mean of the BSA calculated using each equation and the normogram was used to assess influence of BSA on bias. Pure error was calculated as root of the mean of squared deviations (bias). This was done in order to remove any algebraic effects on calculating the mean bias. One way ANOVA was used to compare the BSA assessed by different methods. Statistical analyses were done using NCSS 2000 (Hintze JL, Kayswille, Utah, USA) statistical computer packages.

\section{Results}

Two thousand three hundred and thirty eight girls and 639 boys, between the ages of 5-16 years were assessed. The demographic characteristics of the study population are shown in table 1 . The mean age of the boys was $10.3 \pm 1.47$ years and for girls $11.5 \pm$ 2.47 years.

Table 1

Demographic characteristics of study population

\begin{tabular}{|l|c|c|}
\hline & Female & Male \\
\hline Number & 2338 & 639 \\
\hline Age $(\mathrm{yrs})$ & $11.5 \pm 2.5$ & $10.4 \pm 1.5$ \\
\hline Height $(\mathrm{cm})$ & $141.2 \pm 14.4$ & $138.0 \pm 9.9$ \\
\hline Weight $(\mathrm{kg})$ & $36.4 \pm 12.9$ & $33.9 \pm 11.1$ \\
\hline BMI $\left(\mathrm{kgm}^{2}{ }^{2}\right)$ & $17.8 \pm 4.1$ & $17.5 \pm 4.2$ \\
\hline
\end{tabular}

Table 2 shows the BSA estimations done using the normogram and each of the prediction equations. Highest mean BSA estimation was given by Gehan \& George equation (boys $1.14 \pm 0.20 \mathrm{~m}^{2}$ and girls $1.2 \pm 0.25 \mathrm{~m}^{2}$ ) and lowest by $\mathrm{Yu}$ et al equation (boys $1.08 \pm 0.20 \mathrm{~m}^{2}$ and girls $\left.1.13 \pm 0.25 \mathrm{~m}^{2}\right)$. Estimates correlated highly with BSA assessed by normogram (table 2).

Table 2

Mean BSA assessed by each method and association to criterion method

\begin{tabular}{|l|c|c|c|c|}
\hline & \multicolumn{2}{|c|}{ Female } & Mean \pm SD & $\mathrm{r}^{*}$ \\
\hline Mosteller et al & Mean \pm SD & $\mathrm{r}^{*}$ & $1.13 \pm 0.21$ & 0.9976 \\
\hline DuBios - DuBios & $1.18 \pm 0.26$ & 0.9981 & $1.13 \pm 0.20$ & 0.9913 \\
\hline Boyd & $1.19 \pm 0.25$ & 0.9927 & $1.14 \pm 0.20$ & 0.9974 \\
\hline Gehan and George & $1.19 \pm 0.25$ & 0.9978 & $1.14 \pm 0.20$ & 0.9984 \\
\hline EPA & $1.20 \pm 0.25$ & 0.9987 & $1.14 \pm 0.21$ & 0.9984 \\
\hline Haycock & $1.19 \pm 0.26$ & 0.9988 & $1.12 \pm 0.21$ & 0.9986 \\
\hline Mattar & $1.18 \pm 0.26$ & 0.9989 & $1.12 \pm 0.19$ & 0.9618 \\
\hline Livingston & $1.17 \pm 0.25$ & 0.9697 & $1.13 \pm 0.23$ & 0.9840 \\
\hline Yu et al & $1.18 \pm 0.27$ & 0.9943 & $1.08 \pm 0.20$ & 0.9976 \\
\hline
\end{tabular}

* correlation between BSA assessed by criterion method (normogram) and each prediction technique. All correlations had a significance of $<0.001$.

When the BSA assessed by the normogram and other methods were compared using one way ANOVA, only the BSA assessed by $\mathrm{Yu}$ et al equation did not agree with the normogram value. Figures 1a to $1 \mathrm{~d}$ show the graphs drawn between BSA assessed by normogram and Mosteller and Mattar equations for each gender. It clearly shows that Mosteller equation has higher agreement with the normogram value. 


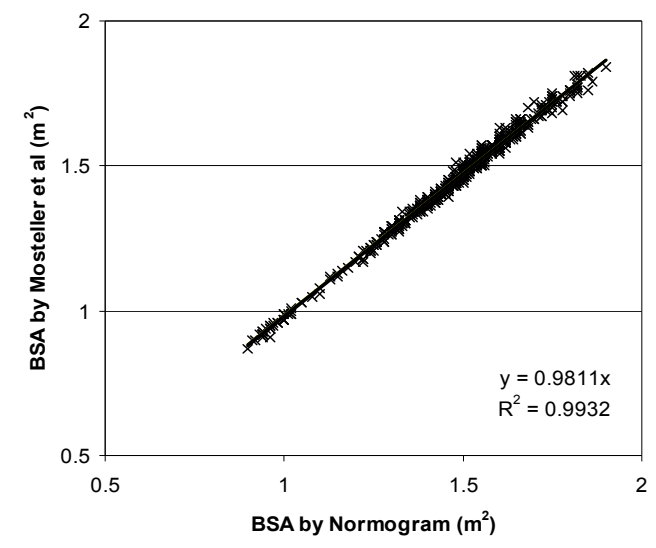

Figure 1a BSA assessed using Mosteller equation in boy

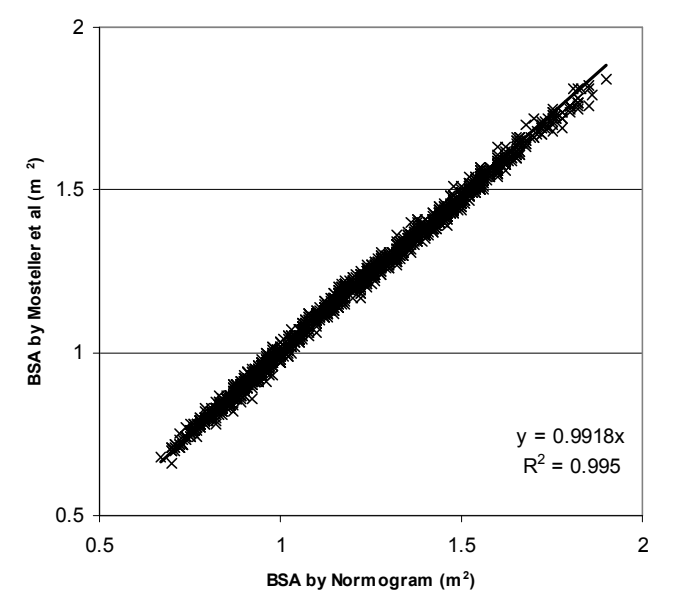

Figure 1b BSA assessed using Mosteller equation in girls

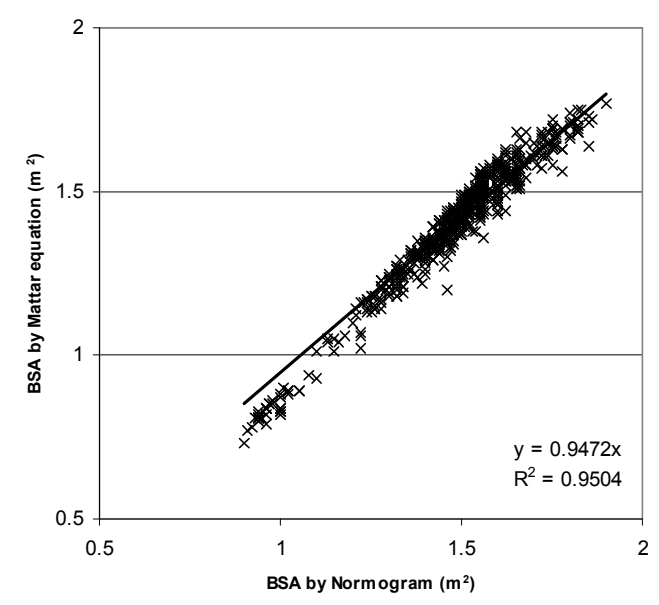

Figure 1c BSA assessed using Mattar equation in boys

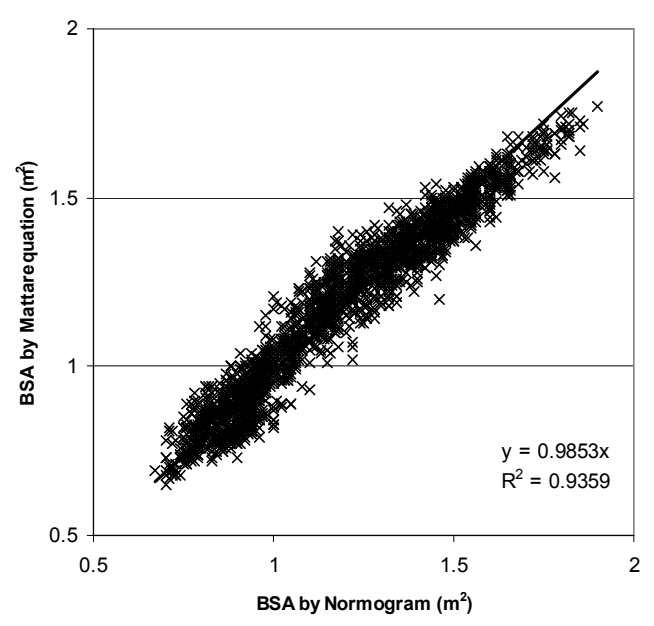

Figure 1d BSA assessed using Mattar equation in girls

Table 3 shows the mean bias for each equation in each gender when compared with the BSA assessed by normogram. Equation of $\mathrm{Yu}$ et al gave the highest bias for both groups (females $0.0604 \mathrm{~m}^{2}$ and males $0.0575 \mathrm{~m}^{2}$ ). The lowest bias in females was given by Boyd equation $\left(0.0017 \pm 0.022 \mathrm{~m}^{2}\right)$ whilst in males it was by DuBios-DuBios equation $\left(-0.0029 \pm 0.037 \mathrm{~m}^{2}\right)$.

Mosteller equation gave relatively low bias (females $0.0077 \mathrm{~m}^{2}$, and males $0.0072 \mathrm{~m}^{2}$ ). Mattar equation in girls gave a bias of $-0.0152 \pm 0.066 \mathrm{~m}^{2}$ and in boys it was $-0.0177 \pm 0.064 \mathrm{~m}^{2}$. However, the correlation between the bias and the mean BSA calculated between the BSA assessed by the equation and nomogram always showed a statistically significant negative association for all equations except for Livingston's equation in girls. This denotes that at extremes of BSA the bias would be more. Most of the associations were negative denoting at lower BSA all equations overestimate and at higher BSA the equations underestimate. Although there is such distortion in the estimation of BSA using prediction equations, the bias values were very minimal and the impact on the biological calculations would be very low. 


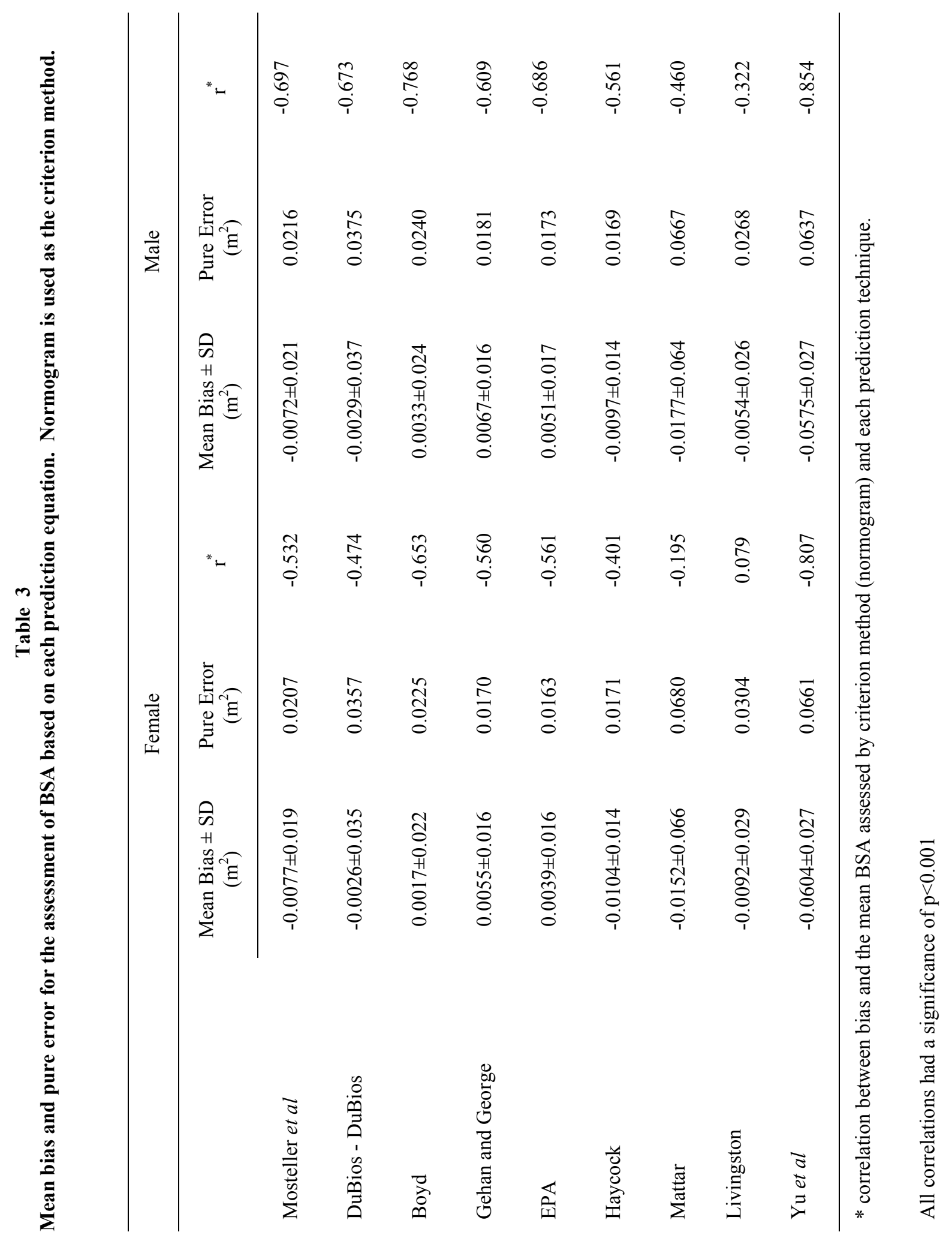


Figure 2a-d shows the Bland Altman plot for Mosteller and Mattar equations.

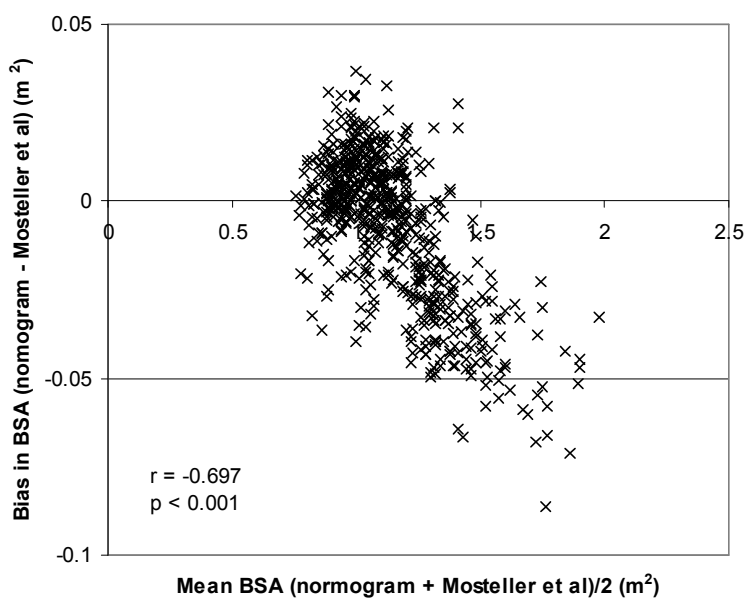

Figure 2a Bland Altman for Mosteller equation in boys

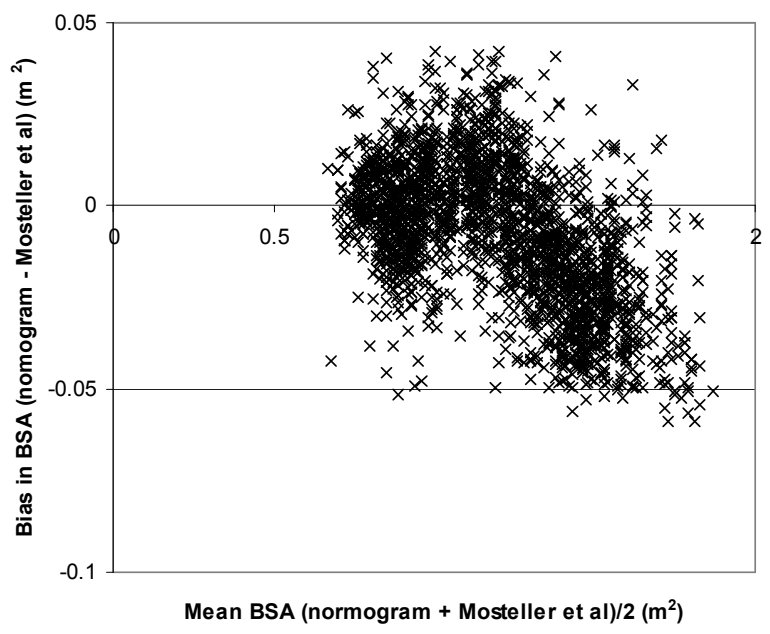

Figure 2b Bland Altman plot for Mosteller equation in girls

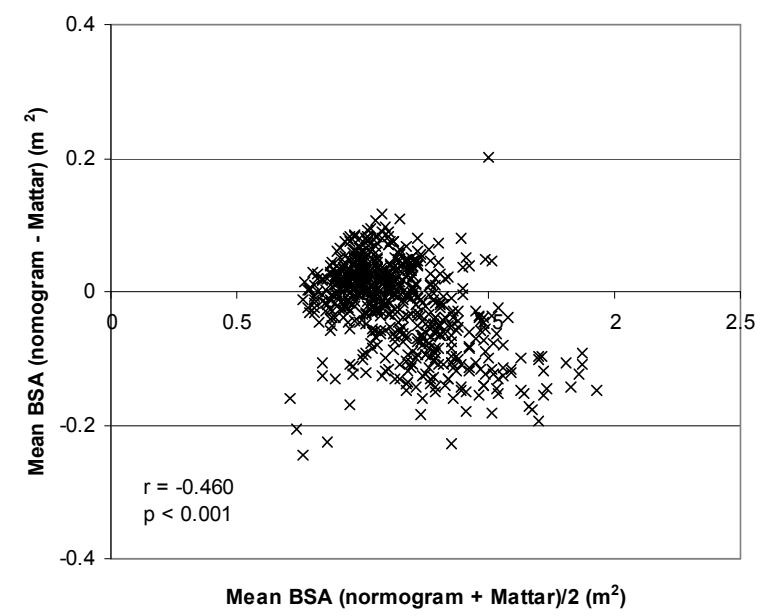

Figure 2c Bland Altman for Mattar equation in boys

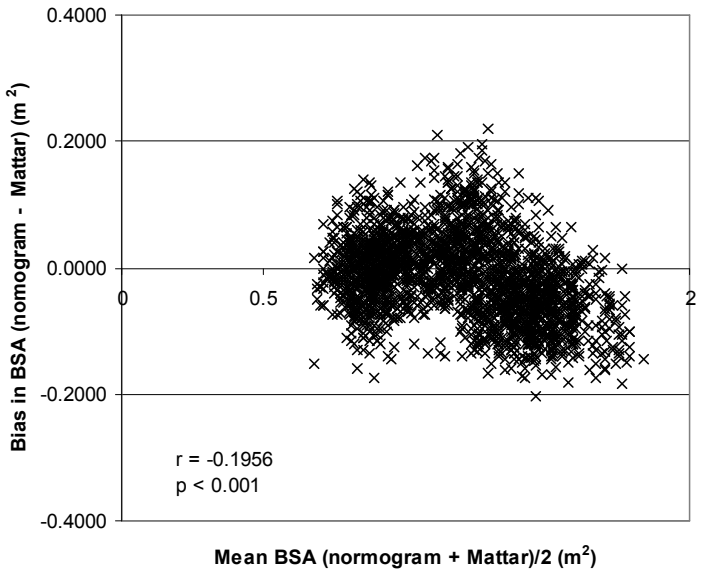

Figure 2d Bland Altman for Mattar equation in girls

In both gender groups the highest pure error was shown by the equation of $\mathrm{Yu}$ et al. Lowest pure error in girls was given by EPA equation and in boys by Haycock equation. When compared the pure error of EPA equation in girls with Mosteller equation there was no statistical difference but there was with Mattar equation. Similarly, when compared, the pure error of Haycock equation in boys with Mosteller equation there was no statistically significant difference but again there was with Mattar equation.

Results were analyzed after stratifying according to age and gender (data not shown). The results did not differ from the main analysis.

\section{Discussion}

Body Surface Area (BSA) is used in day to day clinical practice. Use of the normogram is quite cumbersome as well as it has been shown to cause errors in the assessment ${ }^{1}$. There were many equations in the published literature for the calculation of BSA. However, most of them have complex mathematical calculations which are ideal as research tools but not for routine clinical use. Furthermore, they are derived on adult populations. Mosteller and Mattar equations are two mathematical equations that have very simple calculations and are ideal for day to day clinical work. Former equation had been cross validated on a group of children as well ${ }^{3}$.

This study looked at the possibility of using these equations instead of the Boyd-West normogram in a group of Sri Lankan children. Except for Matter equation, the associations between the BSA assessed by nomogram and each of the equations was very high $(r>0.99)$. Therefore Mosteller equation is ideal for day to day clinical use. The bias given by Mosteller equation is low but it was influenced by the 
extremes of BSA. The pure error of Mosteller equation was not the lowest but it did not have a statistically significant difference from the lowest pure errors. However, before recommending any equation it is useful to consider the impact it would have on clinical use. The maximum bias in estimation would lead to less than $5 \%$ error in determining drug doses which is negligible in day to day clinical practice as dose rounding and inter-patient pharmacokinetics would make a negligible clinical influence

Based on the results of this study Mosteller equation would be a reliable alternative for the calculation of BSA in Sri Lankan children.

\section{References}

1. Briars G, Bailey BJR. Surface area estimation: pocket calculator versus normogram. Arch Dis Child 1994; 70: 2467.

2. Verbraecken J, Heyning PV, Backer WD, Gaal LC. Body surface area in normalweight, over weight, obese adults. A comparison study. Metabolism Clinical and Experimental 2006;55:515-24
3. Lam TKK, Leung DTY. More on simplified calculation of body surface area. $N$ Engl $J$ Med 1988; 318:1130.

4. Mostella RD. Simplified calculation of body surface area. N Engl J Med 1987;317:1098.

5. Bland JM, Altman DG. Statistical methods for assessing agreement between two methods of clinical measurement. Lancet 1986;1(8476):307-10.

Annexure

\begin{tabular}{|c|c|}
\hline \multicolumn{2}{|c|}{ Different equations used in the assessment of } \\
BSA $^{2}$ \\
\hline Mosteller & {$[(\mathrm{H} \times \mathrm{W}) / 3600]^{0.5}$} \\
\hline DuBios - DuBios & $0.00718 \times \mathrm{H}^{0.725} \times \mathrm{W}^{0.425}$ \\
\hline Boyd & $0.0178 \times \mathrm{H}^{0.5} \times \mathrm{W}^{0.484}$ \\
\hline Gehan and George & $0.0235 \times \mathrm{H}^{0.42246} \times \mathrm{W}^{0.51456}$ \\
\hline EPA & $0.0239 \times \mathrm{H}^{0.417} \times \mathrm{W}^{0.517}$ \\
\hline Haycock et al & $0.024265 \times \mathrm{W}^{0.5378} \times$ \\
$\mathrm{H}^{0.3964}$ \\
\hline Mattar & $(\mathrm{H}+\mathrm{W}-60) / 100$ \\
\hline Livingston & $0.1173 \times \mathrm{W}^{0.6466}$ \\
\hline Yu et al & $0.015925 \times(\mathrm{H} \times \mathrm{W})^{0.5}$ \\
\hline
\end{tabular}





